\title{
MOST QUASIDIAGONAL OPERATORS ARE NOT BLOCK-DIAGONAL
}

\author{
DOMINGO A. HERRERO
}

(Communicated by Paul S. Muhly)

\begin{abstract}
The set of all block-diagonal operators is a dense first category subset of the class $(Q D)$ of all quasidiagonal operators. On the other hand, the subset of all irreducible quasidiagonal operators with thin spectra, that are similar to block-diagonal ones, includes a $G_{\delta}$-dense subset of $(Q D)$.
\end{abstract}

C.-K. Fong proved that most normal operators are diagonal, in the sense that the class of all normal operators includes a $G_{\delta}$-dense subset of diagonal ones [1]. By using his results in [6], the author proved that Fong's argument can be modified to show that most quasitriangular operators are triangular and most biquasitriangular operators are bitriangular ("most" in the same sense as above [7]). Here operator means a bounded linear mapping from a complex, separable, infinite dimensional Hilbert space $\mathscr{H}$ into itself; $T \in \mathscr{L}(\mathscr{H})(:=$ the algebra of all operators acting on $\mathscr{H}$ ) is called triangular if it admits a representation as an upper triangular matrix with respect to a suitable orthonormal basis of $\mathscr{H} . T$ is bitriangular if both $T$ and its adjoint $T^{*}$ are triangular (not necessarily with respect to the same basis, of course).

Recall that $T \in \mathscr{L}(\mathscr{H})$ is quasidiagonal if there exists an increasing sequence $\left\{P_{n}\right\}_{n=1}^{\infty}$ of finite rank orthogonal projections such that

$$
P_{n} \rightarrow 1 \text { (strongly) and }\left\|T P_{n}-P_{n} T\right\| \rightarrow 0 \quad(n \rightarrow \infty) .
$$

An operator $B$ is block-diagonal if there exists $\left\{P_{n}\right\}_{n=1}^{\infty}$ as above such that $B P_{n}=P_{n} B$ for all $n=1,2, \ldots$ (and therefore $B=\bigoplus_{n=1}^{\infty} B_{n}$, where $B_{n}=$ $\left.\left(P_{n}-P_{n-1}\right) B \mid \operatorname{ran}\left(P_{n}-P_{n-1}\right), n=1,2, \ldots ; P_{0}=0\right)$.

The results of [1 and $\mathbf{7}]$ might suggest that most quasidiagonal operators (class $(Q D))$ are block-diagonals (class $(B D)$ ). But this is definitely false. Reason. If $\mathscr{K}(\mathscr{H})$ denotes the ideal of compact operators, then $(Q D)+\mathscr{K}(\mathscr{H})=(B D)+$ $\mathscr{K}(\mathscr{H})=(B D)^{-}=(Q D)[3]$. (As usual, the upper bar denotes norm-closure.) P. R. Halmos has shown that the irreducible operators form a $G_{\delta}$-dense subset of $\mathscr{L}(\mathscr{H})$ [2] (see also [10]; $A \in \mathscr{L}(\mathscr{H})$ is irreducible if there is no nontrivial subspace $\mathscr{M}$ of $\mathscr{H}$ such that $A \mathscr{M} \subset \mathscr{M}$ and $A(\mathscr{H} \ominus \mathscr{M}) \subset \mathscr{H} \ominus \mathscr{M})$. Furthermore, Halmos actually proved that given $T$ in $\mathscr{L}(\mathscr{H})$ and $\varepsilon>0$, there exists $K_{\varepsilon} \in \mathscr{K}(\mathscr{H})$, with $\left\|K_{\varepsilon}\right\|<\varepsilon$, such that $T-K_{\varepsilon}$ is irreducible. Since $(Q D)$ is a closed subset of $\mathscr{L}(\mathscr{H})$, invariant under compact perturbations, it is not difficult to check that Halmos's argument also proves that the irreducible quasidiagonal operators form

Received by the editors December $9,1987$.

1980 Mathematics Subject Classification (1985 Revision). Primary 47A66.

Key words and phrases. Quasidiagonal operators, block-diagonal operators, first category subset, $G_{\delta}$-dense subset. 
a $G_{\delta}$-dense subset of $(Q D)$. Since the block-diagonal operators are reducible, we conclude that $(B D)$ is a first category subset of $(Q D)$.

(Incidentally: the class of all normal operators does not have this kind of problem because it is a closed subset of $\mathscr{L}(\mathscr{H})$, disjoint from the class of all irreducible operators, and not invariant under compact perturbations.)

Let $\sigma(T)$ denote the spectrum of $T \in \mathscr{L}(\mathscr{H})$, and let $\sigma_{e}(T)$ denote its essential spectrum, that is, the spectrum of $T+\mathscr{K}(\mathscr{H})$ in the quotient Calkin algebra $\mathscr{L}(\mathscr{H}) / \mathscr{K}(\mathscr{H})$. Recall that $\lambda \in \sigma(T)$ is a normal eigenvalue if $\lambda$ is an isolated point of $\sigma(T)$ and the Riesz spectral invariant subspace $\mathscr{H}(T ; \lambda)$ corresponding to the clopen subset $\{\lambda\}$ of $\sigma(T)$ is finite dimensional. (This is equivalent to saying that $\lambda$ is an isolated point of $\sigma(T) \backslash \sigma_{e}(T)$.) Let $\sigma_{0}(T)$ denote the set of all normal eigenvalues of the operator $T$.

Since $(B D)$ is first category in $(Q D)$, the following is, perhaps, the best possible result that we can expect, along the lines of $[1,7]$.

THEOREM. The subset of all those $T$ in $(Q D)$ such that

(i) interior $\sigma(T)=\phi$;

(ii) $\operatorname{dim} \mathscr{H}(T ; \lambda)=1$ for all $\lambda \in \sigma_{0}(T)$, and $\mathscr{H}=\bigvee\left\{\mathscr{H}(T ; \lambda): \lambda \in \sigma_{0}(T)\right\}$;

(iii) $T$ is irreducible; and

(iv) given $\varepsilon(0<\varepsilon<1)$, there exists $B \in(B D)$ and $K_{\varepsilon} \in \mathscr{K}(\mathscr{H})$, with $\left\|K_{\varepsilon}\right\|<$ $\varepsilon$, such that

$$
T=\left(1+K_{\varepsilon}\right) B\left(1+K_{\varepsilon}\right)^{-1}
$$

is a $G_{\delta}$-dense subset of $(Q D)$.

ProOF. First Step. Let $\left\{P_{n}\right\}_{n=1}^{\infty}$ be a fixed increasing sequence of finite rank orthogonal projections, converging strongly to 1 . Let $(Q D)^{n}$ be the set of all those $T$ in $(Q D)$ satisfying the following conditions

(i) $n$ interior $\sigma(T)$ does not include a disk of radius $1 / n$, and there exists a finite rank Riesz idempotent $E$ (for $T$ ) such that

(ii) $n\left\|E-E^{*}\right\|<1 / n$,

(iii) $)_{n} \sigma(T \mid \operatorname{ran} E)$ consists of exactly rank $E$ distinct normal eigenvalues, and

(iv) $n\left\|P_{n} E P_{n}-P_{n}\right\|<1 / n$.

By using the upper semicontinuity of the spectrum, and the continuity properties of the Functional Calculus, it is not difficult to check that $(Q D)^{n}$ is an open subset of $(Q D)$ (see, e.g., [5, Chapter 1]). Therefore,

$$
(Q D)^{0}=\bigcap_{n=1}^{\infty}(Q D)^{n}
$$

is a $G_{\delta}$ subset of $(Q D)$. A fortiori, so is

$$
(Q D)_{\mathrm{irr}}^{0}:=\left\{T \in(Q D)^{0}: T \text { is irreducible }\right\} .
$$

It is completely apparent that every $T$ in $(Q D)_{\mathrm{irr}}^{0}$ satisfies (i) and (iii). Moreover, since $T$ satisfies (ii) $)_{n}$, (iii) $)_{n}$ and (iv) $)_{n}$ for all $n=1,2, \ldots$, it is not difficult to infer that $T$ admits a sequence $\left\{E_{k}\right\}_{k=1}^{\infty}$ of finite rank Riesz idempotents such that $E_{k} E_{h}=E_{h} E_{k}=E_{k}$ for $1 \leq k \leq h, \sigma\left(T \mid \operatorname{ran} E_{k}\right)$ consists of exactly $\operatorname{rank} E_{k}$ distinct normal eigenvalues of $T$, and

$$
E_{k} \rightarrow 1 \text { (strongly) and }\left\|E_{k}-E_{k}^{*}\right\| \rightarrow 0 \quad(k \rightarrow \infty) .
$$


It readily follows (as in $[\mathbf{1}, \mathbf{7}])$ that $T$ also satisfies (ii).

If $E, F$ are idempotents such that $E F=F E=E$ (so that $\operatorname{ran} E \subset \operatorname{ran} F$ ),

$$
\begin{aligned}
& E=\left(\begin{array}{ccc}
1 & E_{1} & E_{2} \\
0 & 0 & 0 \\
0 & 0 & 0
\end{array}\right) \quad \begin{array}{l}
\operatorname{ran} E \\
\mathscr{H} \ominus \ominus \operatorname{ran} F
\end{array} \\
& F=\left(\begin{array}{ccc}
1 & 0 & F_{1} \\
0 & 1 & F_{2} \\
0 & 0 & 0
\end{array}\right)
\end{aligned}
$$

and we define

$$
V=\left(\begin{array}{ccc}
1 & E_{1} & E_{2} \\
0 & 1 & 0 \\
0 & 0 & 1
\end{array}\right)
$$

then $V$ is invertible,

$$
\begin{aligned}
V^{-1} & =\left(\begin{array}{ccc}
1 & -E_{1} & -E_{2} \\
0 & 1 & 0 \\
0 & 0 & 1
\end{array}\right) \\
V E V^{-1} & =\left(\begin{array}{lll}
1 & 0 & 0 \\
0 & 0 & 0 \\
0 & 0 & 0
\end{array}\right)
\end{aligned}
$$

is the orthogonal projection onto $\operatorname{ran} E$, and

$$
V F V^{-1}=\left(\begin{array}{ccc}
1 & 0 & F_{1}+E_{1} F_{2}-E_{2} \\
0 & 1 & F_{2} \\
0 & 0 & 0
\end{array}\right)=\left(\begin{array}{ccc}
1 & 0 & 0 \\
0 & 1 & F_{2} \\
0 & 0 & 0
\end{array}\right) .
$$

(Indeed, a straightforward computation shows that the condition $E F=F E=E$ is actually equivalent to $E_{2}=F_{1}+E_{1} F_{2}$.)

Thus, if we define

$$
W=\left(\begin{array}{ccc}
1 & 0 & 0 \\
0 & 1 & F_{2} \\
0 & 0 & 1
\end{array}\right)
$$

then $W$ is invertible,

$$
W^{-1}=\left(\begin{array}{ccc}
1 & 0 & 0 \\
0 & 1 & -F_{2} \\
0 & 0 & 1
\end{array}\right)
$$

$W$ and $W^{-1}$ commute with $V E V^{-1}$ (so that

$$
(W V) E(W V)^{-1}=W\left(V E V^{-1}\right) W^{-1}=\left(\begin{array}{lll}
1 & 0 & 0 \\
0 & 0 & 0 \\
0 & 0 & 0
\end{array}\right),
$$

and

$$
(V W) F(V W)^{-1}=\left(\begin{array}{lll}
1 & 0 & 0 \\
0 & 1 & 0 \\
0 & 0 & 0
\end{array}\right)
$$


is the orthogonal projection onto $\operatorname{ran} F$ ); moreover,

$$
\|V-1\|=\left\|\left(E_{1} E_{2}\right)\right\|=\left\|\left(\begin{array}{ccc}
0 & E_{1} & E_{2} \\
-E_{1}^{*} & 0 & 0 \\
-E_{2}^{*} & 0 & 0
\end{array}\right)\right\|=\left\|E-E^{*}\right\|
$$

and

$$
\|W-1\|=\left\|F_{2}\right\|=\left\|\left(\begin{array}{cc}
0 & F_{2} \\
-F_{2}^{*} & 0
\end{array}\right)\right\| \leq\left\|\left(\begin{array}{ccc}
0 & 0 & F_{1} \\
0 & 0 & F_{2} \\
-F_{1}^{*} & -F_{2}^{*} & 0
\end{array}\right)\right\|=\left\|F-F^{*}\right\| .
$$

If, in addition, $E$ and $F$ have finite rank, then $V-1$ and $W-1$ are also finite rank operators.

By passing, if necessary, to a subsequence, we can directly assume that $\left\|E_{k}-E_{k}^{*}\right\|<\varepsilon / 2^{k+2}$. By an obvious inductive argument, we can now construct a sequence $\left\{W_{k}=V_{k} V_{k-1} \cdots V_{2} V_{1}\right\}_{k=1}^{\infty}$ of invertible operators of the form " $1+$ Finite rank", such that

$$
W_{k} E_{j} W_{k}^{-1} \text { is the orthogonal projection onto } \operatorname{ran} E_{j}
$$

$$
\text { for } \begin{aligned}
j=1,2, \ldots, k,\left\|V_{k}-1\right\| & \leq\left\|E_{k}-E_{k}^{*}\right\|<\varepsilon / 2^{k+2}, \\
\left\|W_{k}-1\right\| & =\left\|V_{k} V_{k-1} \cdots V_{2} V_{1}-1\right\| \\
& \leq \sum_{j=1}^{k-1}\left\|V_{k} V_{k-1} \cdots V_{j}-V_{k} V_{k-1} \cdots V_{j+1}\right\| \\
& \leq \sum_{j=1}^{k-1}\left\|V_{k}\right\| \cdot\left\|V_{k-1}\right\| \cdots\left\|V_{j+1}\right\| \cdot\left\|V_{j}-1\right\| \\
& \leq \sum_{j=1}^{k-1}\left\{\prod_{r=j+1}^{k}\left(1+\varepsilon / 2^{r+2}\right)\right\} \varepsilon / 2^{j+2} \\
& \leq \sum_{j=1}^{k-1} \exp \left\{\sum_{r=j+1}^{k} \varepsilon / 2^{r+2}\right\} \varepsilon / 2^{j+2} \\
& <\left(\exp \left\{\sum_{r=2}^{\infty} \varepsilon / 2^{r+2}\right\}\right) \sum_{j=1}^{\infty} \varepsilon / 2^{j+2} \\
& =e^{\varepsilon / 8} \varepsilon / 4<\varepsilon / 2
\end{aligned}
$$

for all $k=1,2, \ldots$, and similarly (for $1 \leq k<h<\infty$ ),

$$
\left\|W_{h}-W_{k}\right\|<e^{\varepsilon / 8} \sum_{j=k}^{h-1} \varepsilon / 2^{j+2}<e^{\varepsilon / 8} \varepsilon / 2^{k+1} \rightarrow 0 \quad(k, h \rightarrow \infty) .
$$

We conclude that $\left\{W_{k}\right\}_{k=1}^{\infty}$ is a Cauchy sequence converging in the norm to an invertible operator $W$ of the form

$$
W=\left(1+K_{\varepsilon}\right)^{-1}
$$

(where $K_{\varepsilon} \in \mathscr{K}(\mathscr{H})$ and $\left\|K_{\varepsilon}\right\|<\varepsilon$ ) such that

$$
\left\{F_{k}=W E_{k} W^{-1}\right\}_{k=1}^{\infty}
$$


is an increasing sequence of finite rank orthogonal projections coverging strongly to the identity. (The above construction was loosely based on [4 and 9].)

Clearly,

$$
B=W T W^{-1}
$$

commutes with $F_{k}$ for all $k=1,2, \ldots$, whence we infer that $B \in(B D)$ and

$$
T=W^{-1} B W=\left(1+K_{\varepsilon}\right) B\left(1+K_{\varepsilon}\right)^{-1}
$$

has the desired form, that is, $T$ also satisfies (iv).

Second Step. It only remains to show that $(Q D)_{\mathrm{irr}}^{0}$ is dense in $(Q D)$.

Claim. $(Q D)^{0} \cap(B D)$ is dense in $(Q D)$.

Let $A \in(Q D)$ and let $\eta>0$ be given. G. R. Luecke proved in [8] that invertible quasidiagonal operators are dense in $(Q D)$. Let $\left\{\lambda_{k}\right\}_{k=0}^{\infty}$ be an enumeration of all those complex numbers whose real and imaginary components are rational numbers $\left(\lambda_{0}=0\right)$. By Luecke's result, there exists $A_{0} \in(Q D)$ invertible, such that $\| A-$ $A_{0} \|<\eta / 2$.

Assume we have already constructed $A_{0}, A_{1}, \ldots, A_{n}$ so that $A_{k}-\lambda_{j}$ is invertible for $0 \leq j \leq k \leq n$, and

$$
\left\|\left(A_{k}-\lambda_{j}\right)^{-1}\right\|^{-1}>\delta_{j}>0
$$

$(k=j, j+1, \ldots, n ; j=0,1,2, \ldots, n)$.

By a formal repetition of Luecke's argument, now we can find $A_{n+1} \in(Q D)$ such that $A_{n+1}-\lambda_{n+1}$ is invertible, $\left\|\left(A_{n+1}-\lambda_{n+1}\right)^{-1}\right\|^{-1}=2 \delta_{n+1}>0,\left\|A_{n+1}-A_{n}\right\|<$ $\eta / 2^{n+1}$, and $A_{n+1}-\lambda_{j}$ is invertible and satisfies

$$
\left\|\left(A_{n+1}-\lambda_{j}\right)^{-1}\right\|^{-1}>\delta_{j}
$$

for $j=0,1,2, \ldots, n$.

Clearly, $\left\{A_{n}\right\}_{n=0}^{\infty}$ is a Cauchy sequence in $(Q D)$ converging to a quasidiagonal operator $C$, which satisfies

$$
\|A-C\|<\eta \quad \text { and }\left\|\left(C-\lambda_{k}\right)^{-1}\right\|^{-1} \geq \delta_{k}>0
$$

for all $k=0,1,2, \ldots$ Hence, $\sigma(C) \cap\left\{\lambda_{k}\right\}_{k=0}^{\infty}=\varnothing$, and therefore

$$
\text { interior } \sigma(C)=\phi \text {. }
$$

According to [3], we can find

$$
D=\bigoplus_{n=1}^{\infty} D_{n} \in(B D)
$$

such that $C-D$ is compact and $\|C-D\|<\eta$. Here

$$
D_{n}=\left(\begin{array}{cccc}
d_{11}(n) & & & * \\
& d_{22}(n) & & \\
& & \ddots & \\
0 & & & d_{r_{n} r_{n}}(n)
\end{array}\right) \begin{aligned}
& e_{1}(n) \\
& e_{2}(n) \\
& \vdots \\
& e_{r_{n}}(n)
\end{aligned}
$$

with respect to some orthonormal basis $\left\{e_{j}(n)\right\}_{j=1}^{r_{n}}$ of the (finite dimensional) subspace of $D_{n}(n=1,2, \ldots)$. 
Since $\sigma_{e}(D)=\sigma_{e}(C)(\subset \sigma(C))$ has empty interior and $D$ is block-diagonal, we can easily check that

$$
\sigma(D)=\sigma_{e}(D) \cup\left(\bigcup_{n=1}^{\infty}\left\{d_{j j}(n): j=1,2, \ldots, r_{n}\right\}\right)
$$

has empty interior. It is obvious that we can find

$$
E=\bigoplus_{n=1}^{\infty}\left(\begin{array}{cccc}
d_{11}^{\prime}(n) & & & * \\
& d_{22}^{\prime}(n) & & \\
& & \ddots & \\
0 & & & d_{r_{n} r_{n}}^{\prime}(n)
\end{array}\right) \begin{aligned}
& e_{1}(n) \\
& e_{2}(n) \\
& \vdots \\
& e_{r_{n}}(n)
\end{aligned}
$$

(The *-entries are exactly the same as for $D_{n}, n=1,2, \ldots$ ) such that

$$
D-E=\bigoplus_{n=1}^{\infty}\left(\begin{array}{cccc}
d_{11}(n)-d_{11}^{\prime}(n) & & 0 \\
& d_{12}(n)-d_{22}^{\prime}(n) & & \\
& & \ddots & \\
0 & & & d_{r_{n} r_{n}}(n)-d_{r_{n} r_{n}}^{\prime}(n)
\end{array}\right) \begin{aligned}
& e_{1}(n) \\
& e_{2}(n) \\
& \vdots \\
& e_{r_{n}}(n)
\end{aligned}
$$

is a trace class diagonal operator such that

$$
\begin{aligned}
\|D-E\| & =\max \left\{\left|d_{j j}(n)-d_{j j}^{\prime}(n)\right|: 1 \leq j \leq r_{n}, n=1,2, \ldots\right\} \\
& \leq \sum_{n=1}^{\infty} \sum_{j=1}^{r_{n}}\left|d_{j j}(n)-d_{j j}^{\prime}(n)\right|=|D-E|_{1}<\eta
\end{aligned}
$$

$\left(|\cdot|_{1}\right.$ denotes the trace norm $)$ and $\sigma(E)$ is the disjoint union of $\sigma_{e}(E)=\sigma_{e}(C)$ and

$$
\sigma_{0}(E)=\bigcup_{n=1}^{\infty}\left\{d_{j j}^{\prime}(n): j=1,2, \ldots, r_{n}\right\} ;
$$

moreover, $E$ can be chosen so that $\mathscr{H}(E ; \lambda)$ has dimension one for each $\lambda \in \sigma_{0}(E)$.

It follows from our construction that

$$
E \in(Q D)^{0} \cap(B D) \text { and }\|A-E\|<3 \eta .
$$

Since $\eta$ can be chosen arbitrarily small, we deduce that $(Q D)^{0} \cap(B D)$ is dense in $(Q D)$.

Therefore, $(Q D)^{0}$ is a $G_{\delta}$-dense subset of $(Q D)$. Since $(Q D)_{\mathrm{irr}}=(Q D) \cap\{T \in$ $\mathscr{L}(\mathscr{H}): T$ is irreducible $\}$ is also a $G_{\delta}$-dense subset, we conclude that

$$
(Q D)_{\mathrm{irr}}^{0}=(Q D)^{0} \cap(Q D)_{\mathrm{irr}}
$$

is a $G_{\delta}$-dense subset of $(Q D)$.

The proof of the theorem is now complete.

REMARK. Since biquasitriangular operators are norm-limits of algebraic operators [5, Chapter 6, 11], and the spectrum of an algebraic operator is finite (and therefore totally disconnected and with empty interior), the same kinds of arguments show that the subsets

$\{T \in \mathscr{L}(\mathscr{H}):(\mathrm{i}) T$ is bitriangular, (ii) $\sigma(T)$ is totally disconnected;

(iii) $\operatorname{dim} \mathscr{H}(T ; \lambda)=1$ for all $\lambda \in \sigma_{0}(T)$ and $\left.\mathscr{H}=\bigvee\left\{\mathscr{H}(T ; \lambda) \lambda \in \sigma_{0}(T)\right\}\right\}$ 
and

$\{T \in \mathscr{L}(\mathscr{H}):$ (i) $T$ is bitriangular; (ii) interior $\sigma(T)=\phi$;

(iii) $\operatorname{dim} \mathscr{H}(T ; \lambda)=1$ for all $\lambda \in \sigma_{0}(T)$ and $\left.\mathscr{H}=\bigvee\left\{\mathscr{H}(T ; \lambda): \lambda \in \sigma_{0}(T)\right\}\right\}$

are $G_{\delta}$-dense subsets of the class of all biquasitriangular operators. (Compare with Theorem 2 and Corollary 9 of [7]!)

This note was written while the author spent part of his sabbatical year at the University of Toronto. The author wishes to thank his colleagues Professors Man-Duen Choi, Chandler Davis and Peter Rosenthal, and to the Department of Mathematics of the University of Toronto for their generous invitation.

\section{REFERENCES}

1. C.-K. Fong, Most normal operators are diagonal, Proc. Amer. Math. Soc. 99 (1987), 671-672.

2. P. R. Halmos, Irreducible operators, Michigan Math. J. 15 (1968), 215-223.

3. __ Ten problems in Hilbert space, Bull. Amer. Math. Soc. 76 (1970), 887-933.

4. D. A. Herrero, Valores límites de integrales multiplicativas de Stieltjes, Rev. Un. Mat. Argentina 24 (1968), 59-64.

5. __, Approximation of Hilbert space operators. Volume I, Research Notes in Math., vol. 72, Pitman, Boston, Mass., London and Melbourne, 1982.

6. _ The diagonal entries in the formula 'quasitriangular-compact=triangular', and restrictions of quasitriangularity, Trans. Amer. Math. Soc. 298 (1986), 1-42.

7. __ Most quasitriangular operators are triangular, most biquasitriangular operators are bitriangular, J. Operator Theory (to appear).

8. G. R. Luecke, A note on quasidiagonal and quasitriangular operators, Pacific J. Math. 56 (1975), 179-185.

9. V. A. Prigorskiř, On similarity of chains of projections in Hilbert space, Mat. Issled. 5 (1970), vyp. 3 (17), 207-209. (Russian)

10. H. Radjavi and P. Rosenthal, The set of irreducible operators is dense, Proc. Amer. Math. Soc. 21 (1969), 256.

11. D. Voiculescu, Norm-limits of algebraic operators, Rev. Roumaine Math. Pures Appl. 19 (1974), 371-378.

Department of Mathematics, Arizona State University, Tempe, Arizona 85287 